\title{
Research and Analysis of Digital Baseband Transmission System Based on MATLAB
}

\author{
Jianwen Chai,Yinghui Nie \\ Physics and Electronics Institute, Hanzhong University, Shaanxi, China
}

\begin{abstract}
This paper mainly studies the basic concept of baseband transmission of digital signal and the transmission process of digital signal baseband transmission and how to design digital baseband transmission system with MATLAB software simulation. This paper first introduces the theoretical basis of this subject, including digital communication, the composition of digital baseband transmission system and digital baseband signal transmission process. Then the paper introduced the characteristics of digital baseband transmission system, including digital PAM signal power density and common line pattern, and by comparing choosing the final bipolar zero return code. Then we introduced the MATLAB simulation software. The conditions of the best reception of the digital baseband signal are described and how the waveform of the baseband signal is observed by an oscilloscope. Finally, according to the basic steps of simulation process, the simulation process of digital baseband transmission system is realized by MATLAB simulation tool, and the system is analyzed.

KEYWORDS: digital baseband transmission system MATLAB computer simulation
\end{abstract}

Citation: Chai JW and Nie YH. Research and Analysis of Digital Baseband Transmission System Based on MATLAB, Journal of Secure Communication and System (2017); 1(1):

*Correspondence to: Jianwen Chai, Physics and Electronics Institute, Hanzhong University, Shaanxi, China, herohere@ gmail.com.

\section{Introduction}

Compared with analog communication, digital communication has many excellent features, its main drawback is that the equipment is complex and requires a larger transmission bandwidth. In recent years, with the advent of large scale integrated circuits, the complexity and technical difficulty of digital systems have been greatly reduced, and the use of high-capacity transmission media such as efficient transmission compression technology and fiber is gradually becoming a solution to the bandwidth problem. As a result, digital transmission is gaining popularity.

Communication principle computer simulation experiment, is the simulation of digital baseband transmission system. The simulation tool is MATLAB programming language. MATLAB is an advanced high-tech programming language, mainly used for numerical calculation and visual graphics processing. It is characterized a high efficient programming tool, integrating numerous functions such as numerical analysis, matrix computing, graphics, image processing and simulation into an extremely interactive environment of pseudo-scientific research, engineering design, and many disciplines. Using MATLAB, digital baseband transmission system can be more comprehensively studied. In order to make the undergraduate students learn communication courses, through the course design we conducted a pilot simulation of the content of the course of communication principles.

\section{Text}

\subsection{Digital baseband transmission system}

The flexibility of digital processing makes digital information transmitted in a digital transmission system either from various digital codes such as computers, televisions and other data terminals, or from digitally processed pulsecode modulation (PCM) signals from analog signals. In principle, the digital information can be directly expressed and transmitted in digital code sequence, but in the actual transmission, depending on the system requirements and channel conditions, generally needs different ways of coding and the use of a set of limited discrete waveforms. These discrete

Copyright (C) 2017 Chai JW and Nie YH. This is an Open Access article distributed under the terms of the Creative Commons AttributionNonCommercial 4.0 International License (http://creativecommons.org/licenses/by-nc/4.0/), permitting all non-commercial use, distribution, and reproduction in any medium, provided the original work is properly cited. 
waveforms may be unmodulated electrical signals or modulated signals. The spectrum occupied by the unmodulated digital signal starts from zero or very low frequency and is called a digital baseband signal. In some wired channels with low pass characteristics, the baseband signal can be transmitted directly without carrier modulation, especially if the transmission distance is not too far. For example, base pulses are transmitted directly in the computer's local area network. This system, which does not directly transmit digital baseband signals without carrier modulation, is called a digital baseband transmission system. And the transmission system including the modulation and mediation process is called a digital bandpass (or band) transmission system.

At present, although the digital baseband transmission system is not as widely used as bandpass transmission, but it is still meaningful for the research of baseband transmission system. This is because, firstly, symmetrical cable constituting short-range data communication system is still widely used in this transmission. Secondly, with the development of digital communication technology, baseband transmission has a rapid development trend, not only for low-speed data transmission, but also for high-speed data transmission. Thirdly, the baseband transmission contains many basic problems of band-pass transmission, that is, many problems of baseband transmission system is also problems to be considered in band-pass transmission system. Lastly, theoretically it can be proved that any one of the band-pass transmission system with linear modulation can be equivalent to a study of baseband transmission system.

The digital baseband transmission system model shown in Figure 1-1, which mainly includes the code converter, send filter, channel, receive filter, equalizer/sampling decision and other parts.

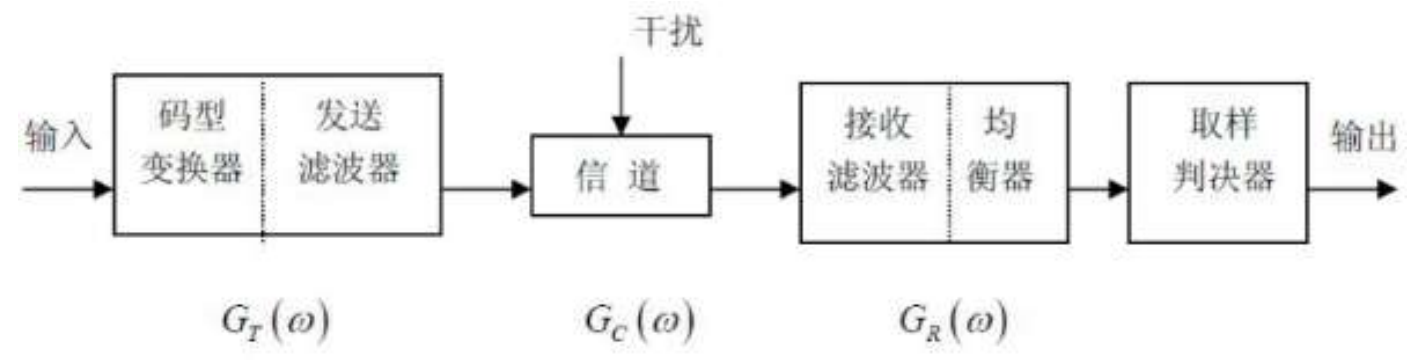

Figure 1. Digital baseband transmission system model

In short, the information of the source is transmitted from the sender through the channel to the receiver at the receiving end in the form of a digital baseband signal. The system is called a digital baseband transmission system.

1) Channel signal generator (transmission filter)

Its function is to generate a baseband signal waveform suitable for channel transmission. Because the input signal is usually generated by the code encoder transmission code, the corresponding basic waveform is usually a rectangular pulse with a wide spectrum. This is not conducive to transmission, hence the transmission filter is used to compress the input signal band, converting the input code into appropriate baseband signal waveform to transmit over the channel.

2) Channel

A channel is a medium that allows baseband signals to pass through, typically wired channels such as twisted pairs, coaxial cables and many more. The transmission characteristics of the channel are generally not satisfying nondistorted transmission conditions and contain additive noise (tn, which can cause the transmission waveform distortion.) The digital baseband transmission system studied in this paper adopts the zero mean white Gaussian noise channel, i.e AWGN channel.

\section{3) Receiving filter}

It is used to receive the signal, as far as possible to filter out channel noise and other interference, and counter balance the channel, so that the output of the baseband waveform is conducive to for sampling decision.

\section{4) Sampling decider}

The sampling decider samples output waveform of the receiving filter at a predetermined time (controlled by the bit timing pulse). When the background of the transmission characteristic is not ideal with noise background, it decides to recover or reproduce the baseband signal.

5) Timing pulse and synchronous extraction

The timing pulse used for sampling is extracted from the received signal by the synchronous extraction circuit. The accuracy of the bit timing pulse will directly affect the decision result. 


\subsection{Digital baseband signal}

\subsubsection{Basic baseband signal waveforms}

The digital baseband signal is an electrical representation of digital information, which can represent the corresponding message code with a different level or pulse. There are many types of digital baseband signals. Here are some basic baseband signal waveforms.

(1) Unipolar waveform

This is one of the simplest baseband signal waveforms. It corresponds to the binary codes ' 1 ' and ' 0 ', respectively, with positive and zero levels, or ' 1 ' and ' 0 ' with pulse, present or absent in one symbol time. The waveform is characterized by no gap between the electrical pulse, the polarity is singular, easy-to-use TTL and CMOS circuit generated. The disadvantages are it is a DC component and requiring transmission lines with DC transmission capacity, which is not suitable for AC coupling long-distance transmission. It is only suitable for internal or very close transmission of the computer.

(2) Bipolar waveform

It denote the ' 1 ' and ' 0 ' of the binary code with positive and negative pulses, respectively. Since the magnitude of the positive and negative levels is equal and the polarity is opposite, when there is no tributary component such as ' 1 ' and ' 0 ', it is advantageous to transmit in the channel and the decision level of the recovery signal at the receiving end is zero in value, which is not affected by changes in channel characteristics. Its anti-interference ability is also strong.

(3) Unipolar zero return waveform

The so-called zero return waveform is its electrical pulse width is less than the symbol width, that is, the signal voltage in a symbol before the end of time always returns to zero level. In general, the zero-shift waveform uses the half-occupied code, that is, the duty ratio $\left(\tau / T_{s}\right)$ of $50 \%$. The non-polar RZ waveform can directly extract the timing information, which is a transition waveform that is often used by other code extraction bit synchronization information. Corresponding to the zero return waveform, the above unipolar waveform and bipolar waveform are non-return to zero waveform, its duty ratio, $\tau / T_{s}$ is $100 \%$.

(4) Bipolar zero return waveform

It is a zero-form of bipolar waveforms. It has both bipolar and zeroed waveforms. Because there is a zero potential interval between adjacent pulses, it is easy for the receiver to recognize the start and end of each symbol, so that both parties can keep the correct synchronization.

(5) Differential waveform

This waveform is represented by the transitions of the level of adjacent symbols and the invariant representation of the message code, irrespective of the potential or polarity of the symbol itself. Since the differential waveform is represented by the relative change of the adjacent pulse level, it is also called the relative code waveform, and the corresponding unipolar or bipolar waveform is the absolute code waveform. The use of differential waveforms to transfer code can eliminate the impact of the initial state of the device, especially in the corresponding modulation system can be used to solve the carrier phase blurring problem.

(6) Multi-level waveform

There are only two levels of the above waveforms, that is, a binary relative code corresponds to a pulse. In order to improve the band utilization, multi-level waveform or multi-value waveform can be used. Since a pulse of multilevel waveform corresponds to multiple binary codes, the bit rate is improved under the same baud rate, so multi-level waveform is widely used in high-speed data transmission system with limited band.

\subsubsection{Common patterns for baseband transmission}

(1) AMI code

The full name of the AMI code is Alternate Mark Inversion code.

Encoding rules: The ' 1 ' code in the symbol sequence is changed to the transmission codes $+1,-1,+1,-1, \ldots$ which are alternately changed in polarity, and the ' 0 ' code in the symbol sequence remains unchanged.

E.g:

Message code: $0110000000110011 \ldots$

AMI code: $0-1+1000000000-1+1000-1+1 \ldots$ 
AMI code corresponding to the waveform is a positive, negative, zero three levels of pulse sequence. It can be seen as a single-pole waveform distortion, that is, ' 0 ' still corresponds to zero level, and ' 1 ' alternately corresponds to positive and negative levels.

Advantages: No DC component. Its low frequency and high frequency components are lesser, energy concentrated at the frequency of $1 / 2$ speed. Codec circuit is simple, and can use the polarity of the exchange of the law to observe the error situation. If it is AMI-RZ waveform, as long as the full-wave undergoes rectification, it can become a unipolar RZ waveform, from which can be extracted timed components.

Disadvantages: When the symbol sequence appears long with ' 0 ', the signal level does not jump for a long time, causing timed signal extraction difficulties.

(2) HDB3 code

HDB3 code's full name is the High Density Bipolar Order 3 code. It is to overcome the transmission waveform appears in the long ' 0 ' code case designed to improve the AMI code.

(1) Check the number of ' 0 ' in the message code. When the number of ' 0 ' is less than or equal to 3 , HDB3 code and AMI code the same.

(2) if there are four or more ' 0 ' in a string, then form every 4 with ' 0 ' into a section, defined as B00V, known as the broken section, where $\mathrm{V}$ is called the damage pulse, and $\mathrm{B}$ the regulation pulse.

(3) $\mathrm{V}$ is the same as the previous non-'0' pulse polarity, and the polarity between adjacent V codes must be alternated. $\mathrm{V}$ has a value of +1 or -1 .

(4) The value of B is optional $0,+1,-1$, so that $\mathrm{V}$ satisfies both of the two requirements in (3) at the same time.

(5) Passport polarity behind the V code should also be alternating.

E.g:

Message code: 100001000011000000001

AMI code: $-100000+1000000-1+1000000000000-1$

HDB3 code: $-10000-\mathrm{V}+10000+\mathrm{V}-1+1-\mathrm{B} 000-\mathrm{V}+\mathrm{B} 000+\mathrm{V}-1$

Where the pulses $\pm V$ and pulses $\pm B$ are the same as the pulse waveform \pm 1 , and the purpose of the $\mathrm{V}$ or $\mathrm{B}$ symbol is to indicate that the nonzero code is transformed from the ' 0 ' of the original code.

HDB3 code coding rules, although are more complex, the decoding is relatively simple. As can be seen from the coding process, each $\mathrm{V}$ code is always the same as its previous non-zero code (including B code). Hence from the received code sequence we can easily find the damage point $\mathrm{V}$ code, so the $\mathrm{V}$ code and its first three codes are ' 0 ' code. We then substitute all the -1 into +1 , the original information code can be restored.

HDB3 code is obvious, making it to be able to retain the AMI code without DC components, to facilitate direct transmission, also overcome the long string 0 (even the number of 0 up to 3). HDB3 code spectrum eliminates DC and very low frequency components, at the same time eliminates the high frequency components in the square wave, making it very suitable for baseband transmission system. Therefore, HDB3 code is currently the most widely used in the actual system code. Although the HDB3 code is better than the AMI code, it still belongs to the 1B1T pattern.

(3) Dual phase code

Dual code is also called Manchester code. It represents the symbol ' 0 ' with a positive and negative symmetrical square wave of a cycle, and uses its inverted waveform to represent the symbol '1'.

Encoding rules: '0' code with '01' two-bit code, '1' code with '10' two-bit code.

E.g:

Message code: 1100101

Dual phase code: 10100101100110

This code has a level transition at the center of each symbol, thus facilitating the extraction of the timing synchronization signal, and the size of the timing component is not affected by the statistical characteristics of the source. In Manchester code, because the positive and negative pulse each half, so there is no DC component, but frequency band occupied has doubled. The Manchester code is suitable for transmission over a short distance of coaxial cable channel. 
(4) Differential biphase code

In order to solve the decoding error caused by the polarity inversion of the biphasic code, the concept of the differential biphase code can be used. The biphase code is synchronized and the code is represented by a level transition in the middle of each symbol duration. In the differential biphase code, the level transitions in the middle of each symbol are used for synchronization, and code can be determined via an extra transition at the beginning of each symbol.

(5) Miller code

Miller code, also known as delay modulation code, is a distortion of the biphase code.

Encoding rules: ' 1 ' code is represented with a symbolic center point jump, that is, ' 10 ' or ' 01 ' . ' 0 ' code has two cases: for a single ' 0 ', the symbol duration does not appear level jump, and with the adjacent symbol does not jump on the border. During continuous ' 0 ', there are two level transition at the boundary of the ' 0 ' code, that is, alternated by ' 00 ' and '11'.

(6) CMI code

CMI code is the abbreviation of the coded mark inversion, similar to the biphase code, it is also a bipolar two-level code.

Encoding rules: ' 1 ' code alternately with ' 11 ' and ' 00 ' two-bit code; ' 0 ' code fixed with ' 01 '.

Figure 2. CMI coding sequence diagram 


\subsection{Experimental principles}

\subsubsection{Digital baseband transmission system model}

Assuming $\left\{a_{n}\right\}$ is the input symbol sequence of the transmit filter, in the case of binary, the value of the symbol $a_{n}$ is 0,1 or $-1,+1$. For the sake of analysis, we represent the baseband signal corresponding to this sequence

$$
d(t)=\sum_{n=\infty}^{\infty} a_{n} \delta\left(t-\boldsymbol{T}_{s}\right)
$$

This signal is a sequence consisting of a unit impulse function $\delta(t)$ at time intervals $T_{s}$, and the intensity of each of them is determined by $a_{n}$. When the transmit filter is energized by $d(t)$, the transmit filter generates a large output signal

Where: '*' is a convolution symbol; $g_{T}(t)$ is a single action of $\delta(t)$ to send the basic waveform, that is, the impulse response of the filter.

The transmission characteristic of the transmission filter is $G_{T}(\omega)$,i.e $g_{T}(t)$, determined by the following equation

$$
g_{T}(t)=\frac{1}{2 \pi} \int_{-\infty}^{\infty} G_{T}(\omega) e^{j \omega t} d \omega
$$

If the transmission characteristic of the re-establishment channel is $C(\omega)$, the transmission characteristic of the reception filter is $G_{R}(\omega)$, the total transmission characteristic of the baseband transmission system shown in Figs. 1-2

$$
H(\omega)=G_{T}(\omega) C(\omega) G_{R}(\omega)
$$

The unit impulse response is

$$
h(t)=\frac{1}{2 \pi} \int_{-\infty}^{\infty} H(\omega) e^{j \omega t} d \omega
$$

$h(t)$ is the single influence under $\delta(t)$, the formation of the output waveform $H(\omega)$. Therefore, under the action of the impulse pulse sequence $d(t)$, the output filter output signal $r(t)$ can be expressed as

$$
r(t)=d(t) * h(t)+n_{R}(t)=\sum_{-\infty}^{\infty} a_{n} h\left(t-\pi_{s}\right)+n_{R}(t)
$$

In the formula, $n_{R}(t)$ is the noise generated by the additive noise $n(t)$ after received by the filter.

The sample decision maker $r(t)$ then makes a sampling decision to determine the transmitted digital information sequence is $\left\{a_{n}\right\}$. For example, in order to determine the value of the kth symbol $a_{k}$, we should first sample at the moment $t=\mathbb{E}_{S}+t_{0}$ to determine the value of $r(t)$ at that point. Hence the formula is

$$
\left.r\left(\mathbb{E}_{s}+t_{0}\right)=a_{k} h\left(t_{0}\right)+\sum_{n \neq k} a_{n} h[k-n) T_{s}+t_{0}\right]+n_{R}\left(\mathbb{E}_{s}+t_{0}\right)
$$

Where $a_{k} h\left(t_{0}\right)$ is the summation of the kth received symbol waveform, which is the basis for the determination of $a_{k} ; \quad$ is the sum of the other symbol waveforms other than the kth symbol at the kth sampling

$$
\left.\sum_{n \neq k} a_{n} h[k-n) T_{s}+t_{0}\right]
$$

time, which is the sum of the current symbol. It plays the role of interference on the decision of $a_{k}$, also known as inter-code crosstalk value, because $a_{n}$ is probability of occurrence, so the inter-code crosstalk value is usually a random variable; $n_{R}\left(\mathbb{E}_{s}+t_{0}\right)$ is the output noise at the sampling moment, it is a random interference, will also affect the correct decision of the k-th symbol. 


\subsubsection{Transmission conditions for inter-code crosstalk}

If there is no intersymbol interference between the symbol transmission rate Rs (Bd), theoretically the minimum system bandwidth required is Rs / $2(\mathrm{~Hz})$. The condition of the minimum system bandwidth is that the system transfer function $\mathrm{H}$ is a rectangular function as shown in 2-5 (b). The impulse response of the system is the Fourier transform of $\mathrm{H}(\mathrm{f})$ to $\mathrm{h}(\mathrm{t})=\operatorname{sinc}(\mathrm{T} / \mathrm{Ts})$, as shown in Figure 2-5 (a) $(\mathrm{Ts}=1 / \mathrm{Rs})$, sinc $(\mathrm{t} / \mathrm{Ts})$ is called the ideal Nyquist pulse.

\section{MATLAB software introduction}

\subsection{Software introduction}

MATLAB is released by the mathworks company of United States, mainly for scientific computing, visualization and interactive programming high-tech computing. It integrates numerical analysis, matrix computing, scientific data visualization, and modeling and simulation of nonlinear dynamic systems into an easy-to-use window interface for scientific research, engineering design, and numerous scientific studies that must be validated. The field provides a comprehensive solution and, to a large extent, out of the traditional non-interactive programming language (such as $\mathrm{C}$, Fortran) editing model, representing the advanced level of today's international scientific computing software.

MATLAB, Mathematica, and Maple are three major math software. It is second to none in numerical applications in mathematical technology applications. MATLAB can perform matrix operations, draw functions and data, implement algorithms, create user interface, and connect other programming language programs. It is mainly used in engineering computing, control design, signal processing and communication, image processing, signal detection, financial modeling design/analysis and other fields.

MATLAB basic data unit is a matrix. Its instruction and expression are commonly used in the form similar to the use in mathematics and engineering. Hence, using MATLAB is much simpler to solve the problem than with C, FORTRAN and other languages, and MATLAB also absorbing the advantages of software such as Maple, making MATLAB a powerful math software. In the new versions, C, FORTRAN, C + +, JAVA support are available and can be directly called. The user can also write their own procedures into the MATLAB function library to facilitate their future call, in addition to many of the MATLAB enthusiasts have prepared some classic procedures, users can download directly can be used. The MATLAB family of products can be used to perform the following tasks:

numerical analysis

Numeric and symbolic calculations

Engineering and Scientific Drawing

Design and simulation of control system

Digital image processing technology

Digital signal processing technology

Communication system design and simulation

MATLAB has a wide range of applications, including signal and image processing, communications, control system design, testing and measurement, financial modeling and analysis, and computational biology and many other applications. The additional toolbox (a separate set of specialized MATLAB functions) extends the MATLAB environment to address specific types of problems in these applications.

In the 1970s, Cleve Moler, director of the Department of Computer Science at the University of New Mexico, wrote the earliest MATLAB in FORTRAN in order to reduce the burden on student programming. In 1984, MathWorks, founded by Little, Moler and Steve Bangert, formally introduced MATLAB to the market. By the 1990s, MATLAB has become the standard software for international control.

\subsection{Features of Matlab language}

1, high programming efficiency

2 , easy to use

3, expansion ability

4 , simple language, rich content

5 , efficient and convenient matrix and array operations 
6 , convenient drawing function

\section{Experimental content}

\subsection{Ideal low-pass characteristics}

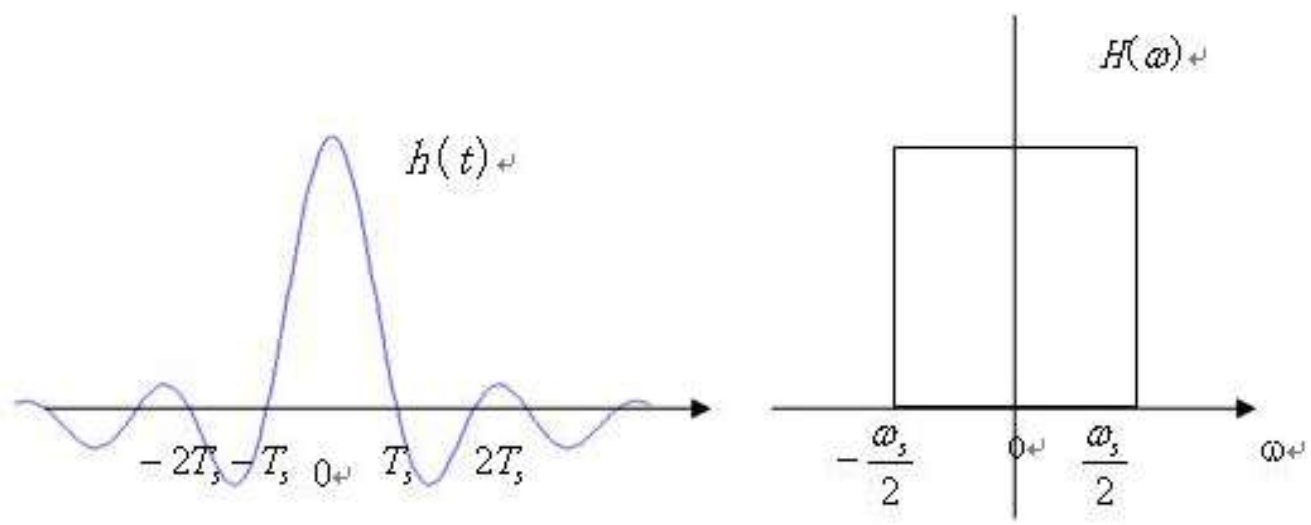

Impulse response

transmission characteristics

Figure 3. Ideal low-pass transmission system characteristics

The main drawbacks of ideal low-pass signals:

A. Engineering and physically difficult to achieve;

B. Tail decay slow (impulse response $\mathrm{h}(\mathrm{t})$ waveform convergence rate is slow, smear to $1 / \mathrm{t}$ rate of attenuation, when timing error will bring more interference) If the sampling time slightly deviated, severe inter-code crosstalk occurs.

\subsection{Cosine roll-off characteristics}

In order to solve the problem of the ideal low-pass characteristic, the edge of the ideal low-pass filter characteristic can be slowly lowered, which is called 'roll-off'. As long as the $H(\omega)$ at amplitude of the center of the roll is in the odd symmetry of the amplitude, it will be able to meet the Nyquist first criteria, achieving inter-coding crosstalk transmission. The transfer function $H(\omega)$, which is rolled by the cosine characteristic, can be expressed as

$$
H(\omega)= \begin{cases}T_{s} & 0 \leq|\omega|<\frac{(1-\alpha) \pi}{T_{s}} \\ \frac{T_{s}}{2}\left[1+\sin \frac{T_{s}}{2 \alpha}\left(\frac{\pi}{T_{s}}-\omega\right)\right] & \frac{(1-\alpha) \pi}{T_{s}} \leq|\omega|<\frac{(1+\alpha) \pi}{T_{s}} \\ 0 & |\omega| \geq \frac{(1+\alpha) \pi}{T_{s}}\end{cases}
$$

Its corresponding $h(t)$ is

$h(t)=\frac{\sin \pi t / T_{s}}{\pi t / T_{s}} \cdot \frac{\cos \alpha \pi t / T_{s}}{1-4 \alpha^{2} t^{2} / T_{s}^{2}}$

In the formula, $\alpha$ is the roll-off coefficient is used to describe the degree of roll-off. It is defined as $\alpha=f_{\Delta} / f_{N}$ 
Where, $f_{N}$ is the Nyquist bandwidth; $f_{\Delta}$ is the amount of expansion beyond the Nyquist bandwidth.

\subsection{Cosine roll-off system based on Matlab procedures and simulation results}

Cosine roll system based on matlab simulation, the source code is as follows:

Ts $=1 ; \%$ sampling interval

$\mathrm{N}=17 ; \%$ Sampling points

$\mathrm{Dt}=\mathrm{Ts} / \mathrm{N} ; \%$ time sampling interval

$\mathrm{Df}=1.0 /(20.0 * \mathrm{Ts})$;

$\mathrm{T}=-10 * \mathrm{Ts}: \mathrm{dt}: 10 * \mathrm{Ts}$;

$\mathrm{F}=-2$ / Ts: df: 2 / Ts;

$\mathrm{A}=[0,0.5,1]$;

For $\mathrm{n}=1$ : length (a)

For $\mathrm{k}=1$ : length $(\mathrm{f})$

If abs $(\mathrm{f}(\mathrm{k}))>0.5 *(1+\mathrm{a}(\mathrm{n})) / \mathrm{Ts}$

$\mathrm{Xf}(\mathrm{n}, \mathrm{k})=0$;

Else if abs (f (k)) <0.5*(1-a (n)) / Ts

$\mathrm{Xf}(\mathrm{n}, \mathrm{k})=\mathrm{Ts}$;

$(\operatorname{Abs}(\mathrm{f}(\mathrm{k}))-0.5 *(1-\mathrm{a}(\mathrm{n})) /(\mathrm{a}(\mathrm{n})+\mathrm{eps}) *(\mathrm{abs}(\mathrm{f}(\mathrm{k}) \mathrm{Ts})))$

End

End

$\mathrm{Xt}(\mathrm{n}): \operatorname{sinc}(\mathrm{t} / \mathrm{Ts}) . *(\operatorname{Cos}(\mathrm{a}(\mathrm{n}) * \mathrm{pi} * \mathrm{t} / \mathrm{Ts})) . /(1-4 * \mathrm{a}(\mathrm{n}) \wedge 2 * \mathrm{t} . \wedge 2 / \mathrm{Ts} \wedge 2+\mathrm{eps}) ;$

End

Subplot (211);

Plot (f, xf);

Axis ([- $\left.\left.\begin{array}{llll}-1 & 1 & 0 & 1.2\end{array}\right]\right)$;

Xlabel ('f / Ts');\% plus x axis instructions

Ylabel ('the spectrum of the raised cosine roll system');\% plus y-axis description

('A $=00^{\prime},{ }^{\prime} \alpha=0.5$ ', ' $\alpha=1$ ');\% plus the legend

Subplot (212);

$\operatorname{Plot}(\mathrm{t}, \mathrm{xt})$;

Axis ([- $\left.\left.\begin{array}{llll}-10 & 10 & -0.5 & 1.1\end{array}\right]\right)$;

Xlabel ('t');\% plus $\mathrm{x}$ axis instructions

Ylabel ('time-domain waveform of the raised cosine roll-off system');\% plus y-axis description

('A $=0$ ', ' $\alpha=0.5$ ', ' $\alpha=1$ ');\% plus the legend

\section{Conclusions}

In the above results we can see that the frequency domain waveform at the center of the roll frequency is at the center of the odd symmetry to meet the Nyquist first criterion. The graph shows that the larger the roll-off coefficient, the greater the amount of expansion beyond the Nyquist bandwidth and the increased bandwidth.

In the time domain waveform, the greater the roll-off coefficient, the faster the run-down attenuation of the waveform, the lower the bit-time accuracy requirements. 
Mathematical analysis shows that the lift cosine roll-off system not only satisfies the transmission condition of codeless crosstalk on the sampling value, but also adds a zero point between the sample values, and its tail attenuation is faster, which is beneficial to reduce the inter-code crosstalk and bit timing error. However, this system occupies twice the bandwidth of the ideal low-pass system, the band utilization rate is $1 B / E I$, half of maximum utilization of the base station system.

The experiment is mainly using MATLAB software to carry out digital baseband communication system simulation. In the whole experiment process, there are several problems.

1, In the beginning I was not very familiar of the overall composition of the system. Later communication with other students I understood more the composition of the entire system, the steps and processes of programming.

2, Because we are unskilled for the use of Matlab software, so there is no simulink simulation. I will continue to study and efforts in the next experiment.

3 , In view of a large program of writing, there will always be problems in the operation, the results do not run out. I started run in different segment, change the mistakes one after another, and later through the efforts of other students, I learned to use breakpoints to find the wrong and single-step operation of the program, which is great help for any future experiments.

4, There are no general concept of the results of the whole experiment before the simulation, leading me difficult to judge correctly on the simulation results are. Because of the lack of knowledge, so that in the process of programming there is misunderstanding. In the future, we need to have a solid foundation and be well aware of the system processes. When we encounter the unknown, first check their own solution on the books, if still unresolved then we will discuss with each other.

Throughout the course design, we have learned a lot of theoretical and practical knowledge, which summarized as follows:

1, Unmodulated digital signal occupied by the spectrum began from zero or very low frequency, known as the digital baseband signal.

2, Without the carrier modulation and direct transmission of digital baseband signal system, known as the digital baseband transmission system. The transmission system including the modulation and mediation process is called a digital bandpass (or frequency band) transmission system.

3, Cosine roll-off system frequency domain waveform in the center of the roll-off section of the odd symmetry characteristics meets the Nyquist first criteria. The greater the roll-off coefficient, the greater the amount of expansion beyond the Nyquist bandwidth and the increase of bandwidth.

4 , The cosine roll-off system $h(t)$ not only satisfies the transmission condition of the inter-code crosstalk on the sampling value, but also adds a zero point between the sampling values, and its tail attenuation is faster, which is beneficial to reduce the impact of inter-code crosstalk and bit timing error.

\section{$\underline{\text { References }}$}

1. Fan Changxin. Principles of Communication (3rd Edition), Electronic Engineering Press.

2. Liu Shutang. Modern communication system (first edition), Xi'an Jiaotong University Press.

3. Lucky R W. Automatic Equalization for Digital Communications. Bell Syst. Tech. J., 1965, (44) 4: 547-588.

4. John G. Proakis. Digital Communications. Third Edition 1995.

5. Ma Hongjie. Data communication. Beijing: China Railway Publishing House, 1995. $37 \sim 40$.

6. Ding Yumei, Gao Xiquan, Peng Xueyu. Digital Signal Processing. Xi'an: Xi'an University of Electronic Science and Technology Press, 1994. $265 \sim 268$.

7. Guo Liyun, Liu Zengji, Wang Xinhai, Zhan Daoyong, Yang Qia. Data transmission. Beijing: People's Posts and Telecommunications Press, 1998. $123 \sim 164$.

8. Shi Yang, Yan Weisheng, Li Jun, Zheng Huiyong. MATLAB language essentials and dynamic simulation tool SIM ULINK. Xi'an: Northwestern Polytechnical University Press, 1998. $67 \sim 81$. 